\title{
UTILIZACIÓN DE CRITERIOS BIOCLIMÁTICOS Y FLORÍSTICOS EN LA SUBDIVISIÓN BIOGEOGRÁFICA DEL SECTOR SUBBÉTICO (PROVINCIA BÉTICA)
}

\author{
Pedro SÁNCHEZ-GOMEZ, Juan F. MOTA POVEDA, \\ Francisco GÓMEZ MERCADO, Francisco SAEZ SOTO
}

\begin{abstract}
RESUMEN. Utilización de criterios bioclimáticos y florísticos en la subdivisión biogeográfica del sector subbético (provincia bética, España). Se han estudiado los distintos subsectores reconocidos hasta el momento del sector Subbético: Subbético-Maginense, Cazorlense, Alcaracense y Subbético-Murciano. Atendiendo al régimen de precipitaciones, se han observado diferencias significativas tanto en las precipitaciones anuales, como en su distribución estacional. En los tres primeros subsectores predominan las de carácter invernal; en el Subbético-Murciano las primaverales-otoñales, con mayor incidencia de las de verano. Asimismo, se han analizado los taxones característicos de cada subsector. Del análisis del conjunto de los datos, se ha detectado una gran afinidad entre los subsectores Cazorlense y Alcaracense considerando este último una variante fría del anterior, por lo que se propone la fusión de ambos en un nuevo subsector Alcaracino-Cazorlense. El subsector Subbético-Maginense, con un alto grado de singularidad, presenta mayor influencia de los sectores béticos colindantes. El subsector Subbético-Murciano engloba los territorios más continentales y de mayor influencia iberolevantina.
\end{abstract}

Palabras clave. Sector Subbético, Bioclimatología, Biogeografía, España.

\begin{abstract}
Use of bioclimatic and floristic criteria for the biogeographical division of Subbético sector (Bética province, Spain). All four subsectors from subbetic Sector have been studied. Significative differences of the rainfall regime have been observed on both annual and seasonal rainfall. SubbeticMaginense, Cazorlense and Alcaracense subsectors show higher winter rainfall, and Subbetic-Murciano subsector has a more important rainfall at spring and autum. Characteristic taxa from each subsector have been analyzed, having been detected a great affinity between Cazorlense and Alcaracense subsectors, being the last one a colder version of the former, for that reason it is proposed the fusion of both into a new Alcaracino-Cazorlense subsector. Subbetic-Maginense subsector, with a high degree of singularity, presents a higher influence from nearby betic sectors. Subbetic-Murciano subsector embraces the most continental ranges, with the greatest iberolevantinian influence.
\end{abstract}

Key words. Subbético Sector, Bioclimatology, Biogeography, Spain.

\section{INTRODUCCIÓN}

El sector Subbético comprende las altas montañas calcáreas del Noreste de la provincia corológica Bética. Rivas-Martínez (1987) propuso 3 subsectores: Subbético-Maginense, Cazorlense y Alcaracense. Con posterioridad dentro del territorio se han realizado estudios parciales sobre la flora y vegetación (Gómez Mercado 1989, Mota 1990, Sánchez-Gómez 
1990), en este último, se da a conocer un subsector adicional denominado SubbéticoMurciano, que corresponde a los territorios orientales de mayor influencia iberolevantina.

Recientemente, se ha publicado un listado de endemismos andaluces que aclara en gran medida el valor diagnóstico de los elementos béticos y subbéticos (Rivas-Martínez et al., 1991).

Partiendo de la configuración establecida en el último trabajo mencionado (RivasMartínez et al. op cit. ), se pretende caracterizar los distintos subsectores, así como establecer sus relaciones a nivel bioclimático y taxonómico. Los análisis a nivel sintaxonómico quedan relegados a estudios posteriores.

\section{MATERIAL Y MÉTODOS}

Los datos climáticos corresponden a diversas estaciones pluviométricas (67) y termopluviométricas (27) suministrados por el I.N.M. Salvo los enclaves con escasas estaciones, tan sólo se han tenido en cuenta aquéllas con una secuencia de años significativa $(>20)$. Para el estudio de las precipitaciones se ha calculado el porcentaje de precipitación estacional respecto al total, sumando las cantidades relativas de Diciembre, Enero, Febrero para invierno (I), y así sucesivamente P, V y O. (tabs. 1, 2; fig. 1). El análisis de la varianza de los datos de la tabla 1 ha sido efectuado con el programa Statgraphics 5.0 y queda reflejado en la figura 1 .

Además, se ha tenido en cuenta el índice To=Precipitación media de la máxima mensual caída en un día / Precipitación total x 100, que resulta ser una medida indirecta de la torrencialidad para cesuras ómbricas de igual rango (tab. 1). Pt es la precipitación media anual en $\mathrm{mm}$.

Respecto a las temperaturas, tan sólo se ha calculado el índice de continentalidad (Ic) en las estaciones termopluviométricas (tab. 1), resultando de la diferencia de las temperaturas medias de los meses más cálido y frío, respectivamente; sin embargo, los resultados no han resultado válidos para un área de estudio local.

Otros índices propuestos por RivasMartínez como el It, Im3 o incluso otros más recientes como Itc, Io, etc, (Rivas-Martínez, 1993) no se han tenido en cuenta debido a la escasez de estaciones termopluviométricas en el área de estudio; no obstante, se sigue la terminología y criterios más usuales respecto a los ombrotipos y termotipos.

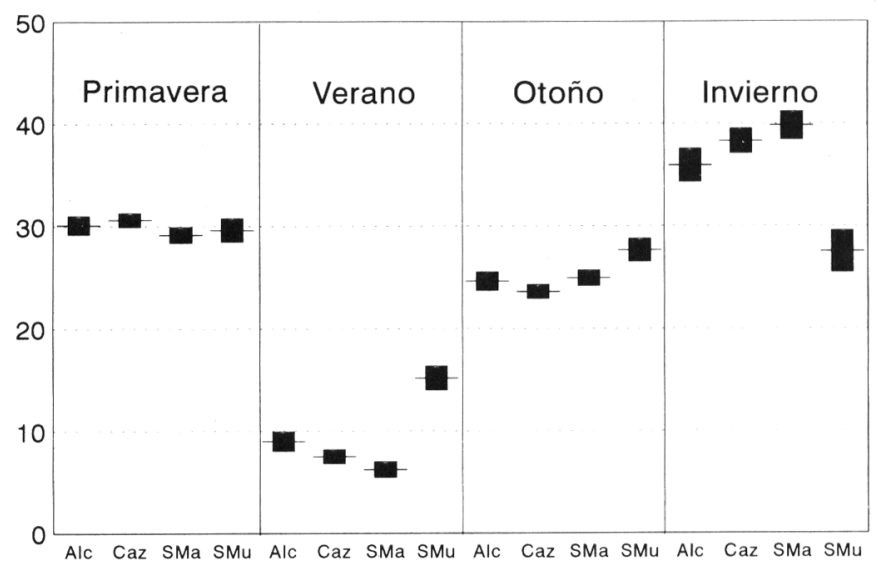

Figura 1. Porcentajes de precipitación estacional con respecto al total. Intervalos de confianza del 95\% para las medias. Seasonal rainfall percentages. $95 \%$ confidence interval for the means. 


\begin{tabular}{|c|c|c|c|c|c|c|c|}
\hline & I & $\mathbf{P}$ & V & $\mathbf{O}$ & To & Pt & Ic \\
\hline Alc Pontones C. H. Segura (J) $1350 \mathrm{~m}$ & 39.3 & 30.3 & 8.0 & 22.4 & 32.6 & 908.7 & 16.6 \\
\hline Alc Yeste, Villar de Tus (Ab) 950 m & 38.6 & 23.2 & 8.5 & 24.3 & 34.5 & 827.4 & \\
\hline Alc Siles, Acebeas (J) $1320 \mathrm{~m}$ & 38.3 & 30.7 & 7.0 & 23.8 & 28.6 & 1166.9 & 17.8 \\
\hline Alc Cañada del Provencio (Ab) $1125 \mathrm{~m}$ & 38.3 & 29.1 & 8.0 & 24.6 & 32.2 & 860.9 & \\
\hline Alc Villapalacios $(\mathrm{Ab}) 836 \mathrm{~m}$ & 37.9 & 29.8 & 7.9 & 24.3 & 32.2 & 618.2 & \\
\hline Alc Bienservida ‘El Batán’ (Ab) $820 \mathrm{~m}$ & 37.8 & 30.5 & 8.7 & 23.1 & 29.3 & 845.8 & \\
\hline Alc Paterna del Madera C. H. Segura (Ab) & 37.4 & 28.0 & 8.8 & 25.7 & 30.4 & 803.1 & 20.6 \\
\hline Alc Segura de la Sierra 'El Campi-' (J) $1410 \mathrm{~m}$ & 37.3 & 29.7 & 7.9 & 25.1 & 30.4 & 987.2 & \\
\hline Alc Arguellite (Ab) $980 \mathrm{~m}$ & 37.1 & 31.1 & 7.6 & 24.2 & 32.7 & 873.1 & 16.9 \\
\hline Alc Bienservida (Ab) $893 \mathrm{~m}$ & 36.8 & 31.7 & 7.2 & 24.2 & 31.5 & 737.4 & \\
\hline Alc Fábricas de Riópar C. H. Segura (Ab) 1000 m & 36.8 & 29.5 & 8.2 & 25.4 & 32.2 & 779.8 & 17.8 \\
\hline Alc $\operatorname{Vianos}(A b) 1117 \mathrm{~m}$ & 36.7 & 31.2 & 8.9 & 23.2 & 31.8 & 789.7 & 21.7 \\
\hline Alc Salobre, Reolid (Ab) $840 \mathrm{~m}$ & 36.7 & 33.0 & 7.7 & 22.6 & 31.5 & 620.1 & \\
\hline Alc Salobre $(A b) 932 \mathrm{~m}$ & 35.9 & 31.9 & 8.7 & 23.5 & 35.8 & 629.5 & \\
\hline Alc Santiago de la Espada 'La To-' (J) 1090 m & 35.7 & 32.3 & 9.2 & 22.9 & 35.1 & 862.9 & \\
\hline Alc Pontones Hoya Espinosilla (J) $1680 \mathrm{~m}$ & 35.2 & 27.2 & 10.0 & 27.7 & 32.4 & 765.5 & \\
\hline Alc Povedilla (Ab) $875 \mathrm{~m}$ & 35.0 & 32.3 & 8.1 & 24.5 & 37.8 & 492.1 & \\
\hline Alc Bogarra, Las Yeguarizas (Ab) $1186 \mathrm{~m}$ & 32.5 & 29.1 & 11.5 & 27.0 & 38.6 & 660.8 & \\
\hline Alc Bogarra $(\mathrm{Ab}) 880 \mathrm{~m}$ & 28.6 & 31.7 & 14.5 & 25.2 & 40.4 & 554.8 & \\
\hline Alc Fuenlabrada (Ab) $1250 \mathrm{~m}$ & 28.0 & 31.1 & 12.4 & 28.5 & 37.6 & 598.5 & \\
\hline Caz Cazorla ‘Nava del Espino’ (J) 1448 m & 43.9 & 29.9 & 5.8 & 20.4 & 29.8 & 1290.2 & \\
\hline Caz Pozo Alcón, El Hornico (J) 1020 m & 43.5 & 27.7 & 5.9 & 22.8 & 35.0 & 799.1 & \\
\hline Caz Peal de Becerro-Fuente Yedra (J) 1220 m & 43.2 & 27.6 & 4.7 & 24.4 & 30.0 & 1105.2 & \\
\hline Caz Santo Tomé 'Fuente Zarza' (J) 1420 m & 43.1 & 30.8 & 4.5 & 21.6 & 28.4 & 1509.8 & \\
\hline Caz Cazorla, Nava de San Pedro (J) 1290 m & 43.1 & 29.2 & 5.5 & 22.1 & 31.2 & 1113.9 & 18.0 \\
\hline Caz La Iruela ‘El Cantalar’ (J) 865 m & 42.6 & 31.1 & 5.0 & 21.3 & 29.2 & 1410.2 & \\
\hline Caz Cazorla, Fuente del Oso (J) $1170 \mathrm{~m}$ & 42.3 & 31.3 & 5.2 & 21.2 & 30.2 & 1278.2 & \\
\hline Caz Cazorla, Vadillo Castril (J) 970 m & 42.3 & 30.5 & 5.6 & 21.6 & 30.8 & 1182.0 & 17.6 \\
\hline Caz Hornos 'Los Casares' (J) $800 \mathrm{~m}$ & 42.2 & 31.5 & 2.7 & 16.8 & 30.8 & 1015.6 & \\
\hline Caz Quesada, Cañada Fuentes'(J) 1474 m & 40.8 & 31.4 & 5.7 & 20.5 & 29.6 & 1435.5 & \\
\hline Caz Castril, Nacimiento, (Gr) $1120 \mathrm{~m}$ & 40.2 & 28.6 & 7.5 & 23.8 & 35.9 & 777.3 & \\
\hline Caz Hornos ‘Cañada Morales’ (J) 780 m & 39.9 & 31.6 & 6.4 & 22.1 & 32.6 & 855.9 & \\
\hline Caz Santiago-Pontones 'Fuente Ro.' (J) 1240 m & 39.8 & 29.9 & 6.6 & 23.7 & 29.5 & 982.9 & \\
\hline Caz Segura de la Sierra 'Morcigui’ (J) 920 m & 39.6 & 31.2 & 6.3 & 22.9 & 30.8 & 776.0 & \\
\hline Caz Segura de la Sierra, El Ojuelo (J) 820 m & 39.0 & 30.2 & 7.0 & 23.7 & 33.5 & 749.2 & \\
\hline Caz Villaverde de Guadalimar (Ab) 799 m & 38.5 & 30.6 & 8.1 & 22.8 & 30.5 & 905.2 & \\
\hline Caz Santiago-Pontones ‘El Queji' (J) 1160 m & 38.3 & 33.1 & 6.3 & 22.2 & 30.3 & 835.0 & \\
\hline Caz Benatae (J) $850 \mathrm{~m}$ & 38.2 & 30.3 & 7.9 & 23.6 & 29.9 & 963.8 & \\
\hline Caz Pantano del Tranco de Beas (J) $600 \mathrm{~m}$ & 38.1 & 32.8 & 6.9 & 22.3 & 31.6 & 849.6 & 18.2 \\
\hline Caz Hornos Segura-Fuente Ganados (J) $1310 \mathrm{~m}$ & 38.0 & 31.1 & 8.7 & 22.2 & 28.9 & 799.0 & \\
\hline Caz Cazorla 'ICONA' (J) $885 \mathrm{~m}$ & 37.8 & 32.5 & 6.4 & 23.2 & 31.0 & 792.4 & 19.0 \\
\hline Caz La Iruela (J) $993 \mathrm{~m}$ & 37.8 & 32.3 & 6.1 & 23.8 & 32.2 & 850.1 & 19.2 \\
\hline Caz Pozo Alcón (J) 877 m & 37.7 & 30.7 & 6.8 & 24.7 & 36.8 & 453.8 & \\
\hline Caz Santiago de la Espada (J) 1328 m & 37.7 & 27.7 & 10.5 & 24.1 & 33.8 & 758.0 & 18.5 \\
\hline Caz Quesada ‘Fuente del Pino' (J) $1400 \mathrm{~m}$ & 36.8 & 31.7 & 6.9 & 24.6 & 32.6 & 946.7 & \\
\hline Caz Siles (J) $826 \mathrm{~m}$ & 36.7 & 31.9 & 7.3 & 24.1 & 32.5 & 785.7 & 21.6 \\
\hline Caz Huescar, San Clemente (Gr) 1043 m & 36.6 & 26.8 & 9.6 & 27.0 & 40.6 & 457.8 & \\
\hline Caz Cazorla, El Chorro (J) $1421 \mathrm{~m}$ & 36.6 & 32.8 & 6.6 & 24.0 & 30.4 & 971.1 & \\
\hline Caz Orcera (J) $796 \mathrm{~m}$ & 36.2 & 32.4 & 8.5 & 23.0 & 33.8 & 625.9 & \\
\hline Caz Huescar, Doctor Jurena $(\mathrm{Gr}) 1100 \mathrm{~m}$ & 33.2 & 29.0 & 11.3 & 26.0 & 44.9 & 419.4 & \\
\hline Caz Huesa, Cerro Miguel (J) 1000 m & 33.2 & 33.5 & 8.2 & 25.1 & 38.0 & 470.4 & \\
\hline Caz Puebla de Don Fadrique 'Los' (Gr) 1198 m & 33.0 & 28.8 & 12.1 & 26.1 & 39.8 & 562.8 & \\
\hline Caz Puebla de Don Fadrique (Gr) 1164 m & 32.0 & 31.2 & 11.7 & 25.2 & 37.9 & 556.7 & \\
\hline
\end{tabular}




\begin{tabular}{|c|c|c|c|c|c|c|c|}
\hline & I & $\mathbf{P}$ & $\mathrm{v}$ & 0 & To & Pt & I \\
\hline Caz Huescar 'ICONA' (Gr) $940 \mathrm{~m}$ & 30.4 & 26.5 & 13.1 & 30.0 & 44.2 & 357.3 & 21.7 \\
\hline Caz Puebla de Don Fadrique ‘Anas' (Gr) 1510 m & 29.7 & 32.0 & 12.9 & 25.3 & 41.6 & 594.9 & \\
\hline SMa Carcabuey, Algar (Co) $740 \mathrm{~m}$ & 46.0 & 28.1 & 5.0 & 20.8 & 31.7 & 795.8 & \\
\hline SMa Almedinilla (Co) $620 \mathrm{~m}$ & 43.5 & 30.1 & 4.0 & 22.4 & 35.1 & 563.4 & 19.0 \\
\hline SMa Illora, televisión (Gr) 1592 m & 43.2 & 22.1 & 7.4 & 27.3 & 34.0 & 568.1 & 17.9 \\
\hline SMa Carcabuey $(\mathrm{Co})$ & 42.6 & 27.9 & 4.5 & 25.0 & 31.8 & 795.2 & 16.7 \\
\hline SMa Alcalála Real, Charilla (J) $920 \mathrm{~m}$ & 42.0 & 27.9 & 6.8 & 23.3 & 31.0 & 681.0 & 17.3 \\
\hline SMa Montillana (Gr) $1022 \mathrm{~m}$ & 41.7 & 27.8 & 5.4 & 25.1 & 28.6 & 609.7 & \\
\hline SMa Montefrío (Gr) 833 m & 41.7 & 26.8 & 5.2 & 26.3 & 32.3 & 641.6 & \\
\hline SMa Valdepeñas de Jaén (J) 927 m & 41.6 & 28.4 & 5.3 & 24.7 & 31.7 & 891.4 & \\
\hline SMa Priego de Córdoba, El Esparra- (Co) 603 m & 41.4 & 28.4 & 5.2 & 25.0 & 34.3 & 707.0 & \\
\hline SMa Bélmez de la Moraleda (J) $887 \mathrm{~m}$ & 41.0 & 27.8 & 8.0 & 23.1 & 38.4 & 612.1 & 19.7 \\
\hline SMa Alcalá la Real (J) 940 m & 40.4 & 29.3 & 6.0 & 24.4 & 29.3 & 699.3 & \\
\hline SMa Noalejo, Hoya Salobral (J) 1140 m & 40.1 & 27.1 & 5.6 & 27.1 & 32.1 & 809.1 & \\
\hline SMa Carcheles, Carchelejo (J) 803 m & 39.9 & 30.2 & 6.7 & 23.1 & 36.6 & 603.9 & \\
\hline SMa Los Villares ‘Riofrío’ (J) 740 m & 39.8 & 30.5 & 5.8 & 23.9 & 32.7 & 838.3 & \\
\hline SMa Torres - C. de A. (J) $888 \mathrm{~m}$ & 39.4 & 28.6 & 7.2 & 29.4 & 35.1 & 695.4 & \\
\hline SMa Iznalloz - Sierra Umbría (Gr) 1060 m & 39.4 & 30.7 & 4.9 & 25.0 & 35.3 & 674.8 & \\
\hline SMa Jodar 'Fuenterroa' $(J) 880 \mathrm{~m}$ & 39.3 & 28.0 & 9.3 & 23.4 & 37.6 & 615.8 & \\
\hline SMa Los Villares - C. de A. (J) $634 \mathrm{~m}$ & 39.3 & 29.0 & 6.0 & 25.7 & 34.3 & 735.0 & \\
\hline SMa Alcalá la Real la Viña (J) 780 m & 39.1 & 29.9 & 4.8 & 26.1 & 34.5 & 506.8 & \\
\hline SMa Priego de Córdoba, Castil de (Co) 640 m & 39.0 & 29.3 & 5.3 & 26.4 & 34.0 & 590.5 & \\
\hline SMa Albánchez de Ubeda (J) $865 \mathrm{~m}$ & 38.9 & 31.3 & 7.3 & 22.4 & 35.3 & 785.0 & \\
\hline SMa Fuente-Tojar (Co) $597 \mathrm{~m}$ & 38.8 & 29.9 & 5.4 & 25.8 & 36.2 & 582.9 & \\
\hline SMa Huelma 'Colegio Nacional' (J) $1002 \mathrm{~m}$ & 38.5 & 30.1 & 7.5 & 23.9 & 31.9 & 591.7 & \\
\hline SMa Jimena (J) $590 \mathrm{~m}$ & 35.8 & 31.4 & 8.1 & 24.7 & 35.5 & 600.3 & \\
\hline SMa Valle de Bedmar y Garcíez (J) $920 \mathrm{~m}$ & 35.4 & 32.0 & 7.8 & 24.8 & 36.5 & 539.9 & \\
\hline SMa Pegalajar - C. de A. (J) 827 m & 34.4 & 32.0 & 7.5 & 26.0 & 36.4 & 548.1 & \\
\hline SMa Jodar - C. de A. (J) 627 m & 33.0 & 32.9 & 6.4 & 27.8 & 37.4 & 479.3 & 20.0 \\
\hline SMu Salto de Miller (J) $709 \mathrm{~m}$ & 36.5 & 29.6 & 10.8 & 23.1 & 35.6 & 625.9 & 19.0 \\
\hline SMu Góntar (Ab) 914 m & 34.3 & 28.3 & 12.4 & 24.9 & 40.1 & 538.8 & 18.8 \\
\hline SMu Yeste C. H. Segura (Ab) 900 m & 31.4 & 30.0 & 12.5 & 26.1 & 40.2 & 554.7 & 17.8 \\
\hline SMu Cañadas de Nerpio (Ab) $1460 \mathrm{~m}$ & 30.3 & 30.6 & 13.8 & 25.3 & 39.0 & 562.5 & \\
\hline SMu Yetas de Abajo (Ab) 1085 m & 29.1 & 30.4 & 14.7 & 25.7 & 40.3 & 510.7 & \\
\hline SMu Nerpio (Ab) $1082 \mathrm{~m}$ & 26.5 & 27.8 & 17.4 & 28.3 & 44.4 & 461.7 & 17.3 \\
\hline SMu Moratalla, Cañada de la Cruz (Mu) 1271 & 25.9 & 28.5 & 17.2 & 28.4 & 42.8 & 409.8 & \\
\hline SMu Benizar $(\mathrm{Mu}) 899 \mathrm{~m}$ & 25.1 & 27.7 & 15.2 & 32.0 & 48.4 & 545.7 & 17.7 \\
\hline SMu Presa del Taibilla $(\mathrm{Ab}) 870 \mathrm{~m}$ & 24.2 & 28.1 & 18.1 & 29.6 & 43.0 & 455.7 & \\
\hline SMu Moratalla, Campo de San Juan (Mu) 1150 m & 23.4 & 31.5 & 14.6 & 30.5 & 49.7 & 427.5 & \\
\hline SMu Moratalla ‘Casas de Alfaro’ (Mu) $1280 \mathrm{~m}$ & 22.1 & 31.2 & 18.6 & 28.0 & 44.8 & 430.4 & 16.4 \\
\hline SMu Moratalla, Bebedor Abajo (Mu) 1085 m & 21.4 & 31.9 & 16.8 & 29.8 & 44.2 & 415.5 & 15.7 \\
\hline
\end{tabular}

Tabla 1. Datos sobre las estaciones termopluviométricas y pluviométricas estudiadas. I: \% precipitaciones Invierno. P: \% precipitaciones Primavera. V: \% precipitaciones Verano. O: \% precipitaciones Otoño. To: Indice de Torrencialidad. Pt: Precipitación anual $(\mathrm{mm})$. Ic: Indice de Continentalidad. Data on thermopluviometric and pluviometric studies stations. I: \% of winter rainfall. $P$ : \% of spring rainfall. V: $\%$ of summer rainfall. O: \% of autumn rainfall. To: Torrentiality Index. Pt: annual rainfall (mm). Ic: Continentality Index. 


\begin{tabular}{lcccc}
\hline & I & P & V & O \\
\hline SMa & 39.8 & 29.2 & 6.2 & 24.9 \\
Caz & 38.3 & 30.6 & 7.5 & 23.5 \\
Alc & 36.0 & 30.1 & 8.9 & 24.6 \\
SMu & 27.5 & 29.6 & 15.2 & 27.6 \\
\hline
\end{tabular}

Tabla 2. Valores medios del porcentaje de precipitaciones estacionales. Mean percentage values for seasonal rainfall.

Para el estudio de los taxones presentes en los distintos subsectores del sector Subbético, se han elegido tan sólo aquellos endemismos de areal bético (se presentan de forma significativa en dos o más sectores de la provincia Bética), o subbético (se presentan en uno o más subsectores del sector Subbético) (tabs.3-5); de este modo, se pretende resaltar el carácter bético, subbético e individual de cada subsector de partida.

El estudio de los datos sobre la presencia o ausencia de taxones béticos y subbéticos en cada subsector, se ha realizado mediante el análisis de componentes principales con el programa Statgraphics 5.0; de este modo, se ha establecido el grado de afinidad entre los distintos subsectores. Los datos han sido ponderados según la escala 1-9, en la que el valor 9 corresponde a endemismo subbético presente en 1 subsector; 8: endemismo subbético presente en 2 subsectores, ...., 5: endemismo bético presente exclusivamente en 1 subsector, 4: endemismo bético presente en 2 subsectores y así sucesivamente hasta el valor 1: endemismo bético presente en los 4 subsectores y que sobrepasa el área bética (señalado con una flecha), es decir, que con carácter puntual puede presentarse en zonas adyacentes a la provincia Bética, en comunidades vegetales que no se consideran de óptimo bético (tabs.3,4 y 5; fig. 2).

Entre las principales obras que se han tenido en cuenta para elaborar las tablas 3-5, además de las que ya se han referido en la introducción, destacan: Castroviejo et al. (1986-1994), Charpin \& Fernández Casas
(1975,1978), Gómez-Mercado \& Valle (1990), Herranz (1984), Herranz et al. (1993), Mota et al. (1991, 1993), Nieto Caldera et al. (1991), Sánchez-Gómez \& Alcaraz (1992, 1993), Sánchez-Gómez et al. (1991,1992), Valle et al. (1989), Güemes et al. (1994), Escudero \& Pajarón (1994). Algunos datos corológicos relativos a la comarca de Yeste han sido facilitados por López Vélez (com. pers.).

Para reforzar la identidad de cada uno de los subsectores se alude a los rasgos que, desde el punto de vista de la vegetación, se dan en cada uno de ellos. Por este motivo se mencionan sintaxones en la descripción de los subsectores, de acuerdo con la propuesta metodológica de la escuela sigmatista de Zürich-Montpellier.

La nomenclatura taxonómica con autoría completa, así como de los sintaxones viene reflejada en las referencias bibliográficas anteriormente mencionadas.

\section{RESULTADOS Y DISCUSIÓN}

Del estudio de los máximos estacionales, (tab. 1,2; fig. 1) se desprende que el mayor valor de I corresponde al subsector SubbéticoMaginense (SMa), seguido del Cazorlense (Caz) y Alcaracense (Alc), aunque estos dos últimos subsectores, presentan algunas estaciones más orientales con I inferior. El subsector Subbético-Murciano (SMu) presenta un régimen marcadamente iberolevantino con valores de $\mathrm{I}=20-30$, y $\mathrm{O}$ algo mayor, a excepción de las estaciones más occidentales. A su vez, el mayor valor de I suele estar relacionado con un ombroclima más lluvioso que va del húmedo en las zonas altas cazorlenses, alcaracenses e incluso subbético-maginenses, hasta el seco, que es dominante en la parte oriental del sector afectada por el efecto Foehn. Por el contrario, estos enclaves suelen tener altos valores de V, que puede llegar incluso a 18 , tal como ocurre en algunas estaciones subbético-murcianas (fig. 2). Este hecho explicaría la presencia de ciertos 
taxones iberolevantinos (Sánchez-Gómez et al. 1992) e incluso la de otros elementos aparentemente más exigentes en humedad, por lo que podemos considerar a estas estaciones, en cierto modo menos mediterráneas; en el extremo opuesto se sitúa el sector SubbéticoMaginense.

El valor de To para iguales cesuras ómbricas es claramente superior en las estaciones orientales, lo que da a entender una mayor torrencialidad de los enclaves con influencia iberolevantina, marcada sobre todo al final del verano y otoño.

Teniendo en cuenta las temperaturas, el valor de Ic no representa los valores a priori esperados, que serían superiores en el subsector Subbético-Murciano. Aunque no se reflejan aleatoriamente en las escasas estaciones termopluviométricas, los pisos bioclimáticos representados van del mesomediterráneo cálido de los subsectores Subbético-Maginense y Cazorlense, hasta el oromediterráneo de todas las altas montañas de los cuatro subsectores.

Del análisis del elemento florístico (fig. $2 ;$ tabs. 3, 4 y 5) se observa una clara afinidad entre los subsectores Cazorlense y Alcaracense, con cierta cercanía hacia el SubbéticoMurciano, el más empobrecido. El subsector Subbético-Maginense constituye un enclave con gran número de elementos béticos, pero muy alejado del resto, probablemente debido a su mayor aislamiento geográfico al quedar claramente separado del resto por la depresión del Guadiana Menor.

El análisis de los factores representados en la figura 2 muestra como el primero de ellos coincide en gran medida con los «endemismos béticos», ya que situa en un extremo al subsector SMa -extraordimariamente rico en endemismos béticos no presentes en los otros subsectores aquí tratados-frente al resto. El segundo factor expresa con bastante exactitud el gradiente de «endemismos subbéticos», por lo que coloca a un lado el subsector Caz y en el otro el SMu, mientras que resultan intermedios los otros dos.

\section{CONCLUSIONES}

El conjunto de los datos aportados, revela que el grado de afinidad bioclimática y florística de los subsectores Cazorlense y Alcaracense es manifiesta. Se propone la fusión de ambos subsectores, que pasarían a formar parte de un sólo subsector AlcaracinoCazorlense. La variabilidad de dichos subsectores deberá ser recogida en unidades biogeográficas de rango inferior una vez sean realizados estudios más exhaustivos. De este modo el encuadre biogeográfico propuesto del sector Subbético queda del siguiente modo: (mapa 1):

Reino Holártico

Región Mediterránea

Subregión Mediterráneo Occidental

Superprovincia Mediterráneo-Iberoatlántica Provincia Bética Sector Subbético Subsector Subbético-Maginense Subsector Alcaracino-Cazorlense Subsector Subbético-Murciano

La descripción somera de cada subsector es la siguiente:

\section{Subsector Subbético-Maginense}

Entre las sierras más importantes de este subsector, por extensión y altitud, se encuentran la $S^{a}$ de Mágina, $S^{\mathbf{a}}$ Harana, Montes Orientales y las Sierras Subbéticas cordobesas (Rute, Horconera, etc.). Se trata de un territorio que incluye diversos macizos más o menos dispersos, rodeados por depresiones que enlazan con las pertenecientes a los sectores Hispalense y Guadiciano-Bacense. De esta forma casi se interrumpe la continuidad con el resto del sector Subbético, debido a la depresión del Guadiana Menor. Presenta también una amplia zona de contacto con el sector Malacitano-Almijarense y, en menor medida, con el Rondeño. Esta posición de vecindad estrecha con unidades corológicas no 
Allium chrysonemum Stearn

Andryala agardhii Haenseler ex DC.

Anthyllis rupestris Cosson

Antirrhinum graniticum subsp. boissieri (Rothm.) Valdés

A. subbaeticum J.Güemes, Mateu \& Sánchez-Gómez

Aquilegia pyrenaica subsp. cazorlensis (Heywood) Galiano \& R.-Martínez

Arenaria alfacarensis Pamp.

A. caesia (Boiss.) Rivas-Martínez, Asensi, Molero Mesa \& Valle

A. modesta subsp. tenuis (Gay) G. López \& Nieto Feliner

A. murcica (Font Quer) Rivas-Martínez, Asensi, Molero Mesa \& Valle

A. tomentosa Willk.

Armeria bourgaei Boiss. ex Merino

A. villosa subsp. alcaracensis Nieto Feliner

A. villosa subsp. longiaristata (Boiss. \& Reuter) Nieto Felíner

Astragalus sempervirens subsp. giennensis (Heywood) Malagarriga

Biarum carratracense (Haenseler) Font Quer

Bupleurum bourgaei Boiss. \& Reuter in Boiss.

Carduncellus hispanicus subsp. macrocephalus (Cuatrec.)

Rivas-Martínez, Asensi, Molero Mesa \& Valle

Carlina baetica (Fernández Casas \& Leal) Fernández Casas

Centaurea boissieri subsp. prostrata (Cosson) Dóstal

C. granatensis Boiss. ex DC.

C. jaennensis Degen \& Debeaux in Degen

C. monticola Boiss. ex DC. subsp. monticola

C. pauneroi Talavera \& Muñoz

Cephalaria linearifolia Lange

Cirsium gregarium Boiss. ex Willk. in Willk \& Lange

Cirsium rosulatum Talavera \& Valdés

Coincya rupestris Porta \& Rigo ex Rouy subsp. rupestris

Convolvulus boissieri Steudel

Crepis granatensis (Willk.) Blanca \& Cueto

C. oporinoides Boiss. ex Froelich in DC.

Cynara baetica (Sprengel) Pau

Daphne oleoides subsp. nevadense (Pau) Rivas-Martínez

Delphinium emarginatum subsp. nevadense (Kunze) Blandré \& Molero

Dianthus subbaeticus Fernández Casas

Erodium astragaloides Boiss. \& Reuter

E.cazorlanum Heywood

Erysimum cazorlense (Heywood) Holub

E. fitzii Polatschek

E. myriophyllum Lange

E. popovii Rothm.

Festuca cordubensis Devesa

F. reverchonii Hackel

Fumana baetica J.Güemes

Fumana paradoxa Heywood

Galium erythrorrhizon Boiss. \& Reuter

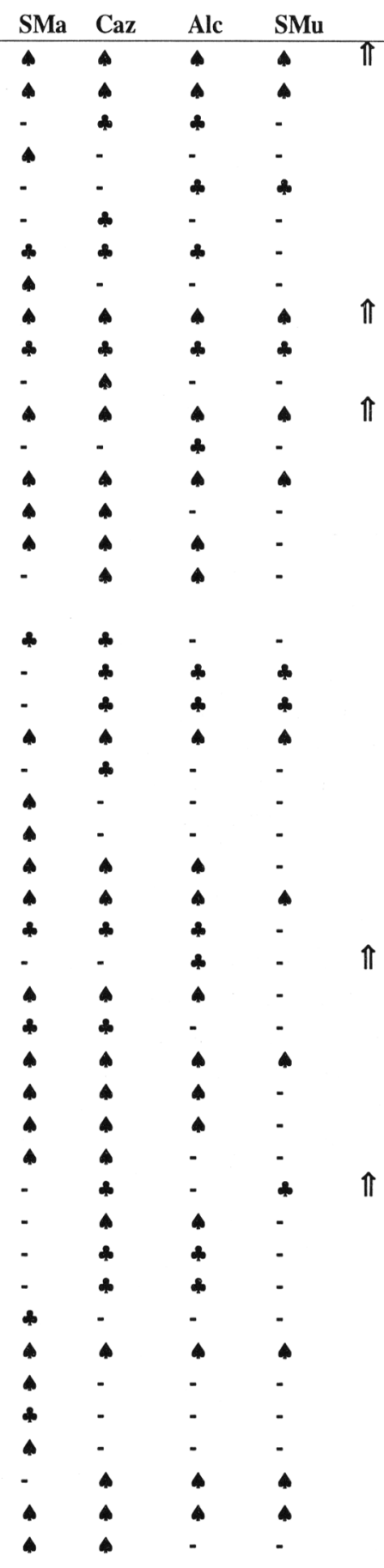




\begin{tabular}{|c|c|c|c|c|c|}
\hline & SMa & $\mathrm{Caz}$ & Alc & SMu & \\
\hline G. rosellum (Boiss.) Boiss. \& Reuter & 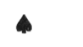 & 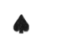 & - & - & \\
\hline Geranium cazorlense Heywood & - & 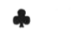 & $\cdot$ & $\cdot$ & \\
\hline Globularia spinosa L. & ↔ & 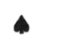 & 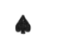 & $\bullet$ & \\
\hline Gypsophila monserratii Fernández Casas & - & 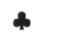 & 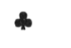 & + & $\Uparrow$ \\
\hline Helianthemum frigidulum Cuatrec. & + & - & - & - & \\
\hline H. viscidulum subsp. viscaroides (Hervier) Guinea \& Heywood & - & $\bullet$ & - & $\cdot$ & \\
\hline \multicolumn{6}{|l|}{ Helictotrichon filifolium subsp. cazorlense (Romero Zarco) } \\
\hline Rivas-Martínez, Asensi, Molero Mesa \& Valle & 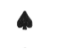 & $\bullet$ & 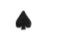 & $\cdot$ & \\
\hline H. sarracenorum (Gand.) J. Holub & 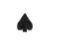 & - & - & $\cdot$ & \\
\hline Herniaria baetica Boiss. \& Reuter & 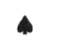 & 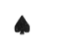 & - & - & \\
\hline Hippocrepis squamata subsp. eriocarpa (Boiss.) Nyman & ↔ & 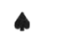 & 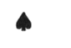 & $\bullet$ & \\
\hline Hormathophylla baetica P. Küpfer & - & 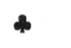 & - & - & \\
\hline H. cadevalliana (Pau) T.R. Dudley & & 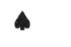 & - & - & \\
\hline H. longicaulis (Boiss.) Cullen \& T.R. Dudley & $\leftrightarrow$ & $\leftrightarrow$ & $\leftrightarrow$ & $\bullet$ & \\
\hline H. reverchonii (Degen \& Hervier) Cullen \& T.R. Dudley & - & 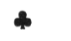 & - & - & \\
\hline Hypochoeris rutea Talavera & 4 & - & - & - & \\
\hline Iberis nazarita Moreno & $\bullet$ & - & - & - & $\Uparrow$ \\
\hline \multicolumn{6}{|l|}{ Jasione crispa subsp. segurensis Mota, Diaz de la Guardia, } \\
\hline Gómez-Mercado \& Valle & $\cdot$ & + & \& & - & \\
\hline Jurinea fontqueri Cuatrec. & + & - & - & - & \\
\hline Lactuca perennis subsp. granatensis Charpin \& Fernández Casas & - & 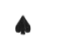 & 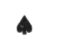 & 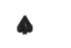 & \\
\hline Leontodon boryi Boiss. ex DC. & - & 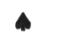 & - & - & \\
\hline \multicolumn{6}{|l|}{ Leucanthemopsis spathulifolia (Gay) Rivas-Martínez, Asensi, } \\
\hline Molero Mesa \& Valle & - & 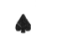 & 4 & $\leftrightarrow$ & \\
\hline Linaria anticaria Boiss. \& Reuter & 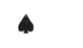 & ๑ & 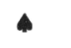 & $\bullet$ & \\
\hline L. lilacina Lange & $\leftrightarrow$ & 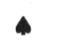 & 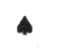 & - & \\
\hline Lithodora nitida (Ern) R. Fernandes & 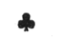 & - & - & - & \\
\hline Melica bocquetii Talavera & $\$$ & 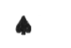 & 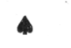 & ^ & \\
\hline Merendera androcymbioides Valdés & $\bullet$ & - & - & - & \\
\hline Moehringia intricata subsp. giennensis D. Guardia, Mota \& Valle & - & + & 4 & 4 & $\Uparrow$ \\
\hline Moricandia moricandioides subsp giennensis Valdés Bermejo & $\bullet$ & 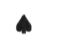 & - & - & \\
\hline Muscari cazorlanum Soriano, Rivas Ponce, Lozano \& Ruiz-Rejón & - & + & - & - & \\
\hline Narcissus assoanus subsp. praelongus A. Barra \& G.López & $\bullet$ & - & - & - & \\
\hline N. bugei (Fernández Casas) Fernández Casas & 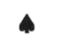 & 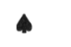 & $\leftrightarrow$ & - & \\
\hline N. longispathus Pugsley & 4 & + & + & - & \\
\hline N. nevadensis Pugsley & - & - & $\leftrightarrow$ & - & \\
\hline Nepeta granatensis Boiss. & - & - & ↔ & - & \\
\hline N. hispanica Boiss. \& Reuter in Boiss & - & $\leftrightarrow$ & - & - & \\
\hline N. tuberosa subsp. gienensis (Degen \& Hervier) Heywood & ↔ & 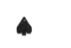 & 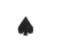 & - & \\
\hline Paronychia kapela subsp. baetica Küfer & - & 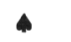 & - & $\leftrightarrow$ & \\
\hline Pinguicula vallisneriifolia Webb & - & 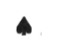 & 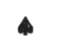 & - & \\
\hline Potentilla petrophila Boiss. & - & 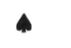 & 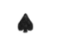 & 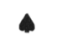 & \\
\hline P. reuteri Boiss. & 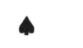 & 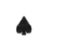 & - & - & \\
\hline Pterocephalus spatulatus (Lag.) Greuter & 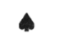 & 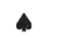 & 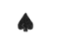 & $\bullet$ & \\
\hline Ptilostemon hispanicus (Lam.) Greuter & 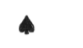 & $\bullet$ & 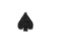 & $\bullet$ & \\
\hline Ranunculus malessanus Degen \& Hervier & - & + & + & - & \\
\hline Reseda phyteuma subsp. rupestris (Lange) Aránega \& Pajarón & $\Delta$ & 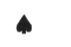 & 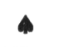 & $\bullet$ & \\
\hline
\end{tabular}




\begin{tabular}{lllll}
\hline SMa & Caz & Alc & SMu \\
\hline
\end{tabular}

Salvia blancoana Webb \& Heldr.

S. oxyodon Webb \& Heldr.

S. phlomoides subsp. boissieri (Noe) Rosúa \& Blanca

Santolina elegans Boiss. in DC.

Sarcocapnos baetica (Boiss. \& Reuter) Nyman subsp. baetica

$S$. baetica subsp. integrifolia (Boiss.) Nyman

S. baetica subsp. ardalii López-Vélez

S. pulcherrima Morales \& Romero-García

Satureja intricata Lange subsp. intricata

Saxifraga camposii Boiss. \& Reuter subsp. camposii

S. erioblasta Boiss. \& Reuter in Boiss.

S. rigoi Porta

Scabiosa andryalifolia (Pau) Devesa

Scilla paui Lacaita

S. reverchonii Degen \& Hervier

Scorzonera albicans Cosson

$S$. reverchonii Debeaux ex Hervier

Scutellaria orientalis subsp. hispanica (Boiss.) Greuter \& Burdet

Senecio pyrenaicus subsp. granatensis (Boiss. ex DC.) R.-Martínez

Senecio quinqueradiatus Boiss. ex. DC.

Seseli montanum subsp. granatensis (Willk.) Pardo

Sideritis carbonellis Socorro

S. laxespicata (Degen \& Debeaux) Socorro, I.Tárrega \& Zafra

Silene germana Gay in Cosson

Sisymbriella aspera subsp. pseudoboissieri (Degen) Heywood

Sisymbrium arundanum Boiss.

Solenanthus reverchonii Degen

Succisella andreae-molinae Escudero \&Pajarón

Teucrium leonis Sennen

T. lerrouxii Sennen

T. luteum subsp. contortostylum (Sennen) Navarro \& Rosúa

T. similatum Navarro \& Rosúa

Thalictrum speciosissimum subsp. albini (Pau) P.Montserrat

Thymelaea granatensis subsp. glauca (Talavera \& Muñoz) R.-Martínez

T. granatensis (Pau) Pau ex Lacaita subsp. granatensis

Thymus granatensis Boiss.

T. x hyeronymi Sennen (Th. mastichina x Th.gadorensis)

T. orospedanus Huguet del Villar

T. x mixtus Pau (Th.mastichina x Th. orospedanus)

T. sabulicola Cosson

T. funkii subsp. burilloi Sánchez-Gómez, Sáez \& Alcaraz inéd

Verbascum hervieri Degen

Vicia glauca subsp. giennensis (Cuatrec.) Blanca \& Valle

Viola cazorlensis Gand

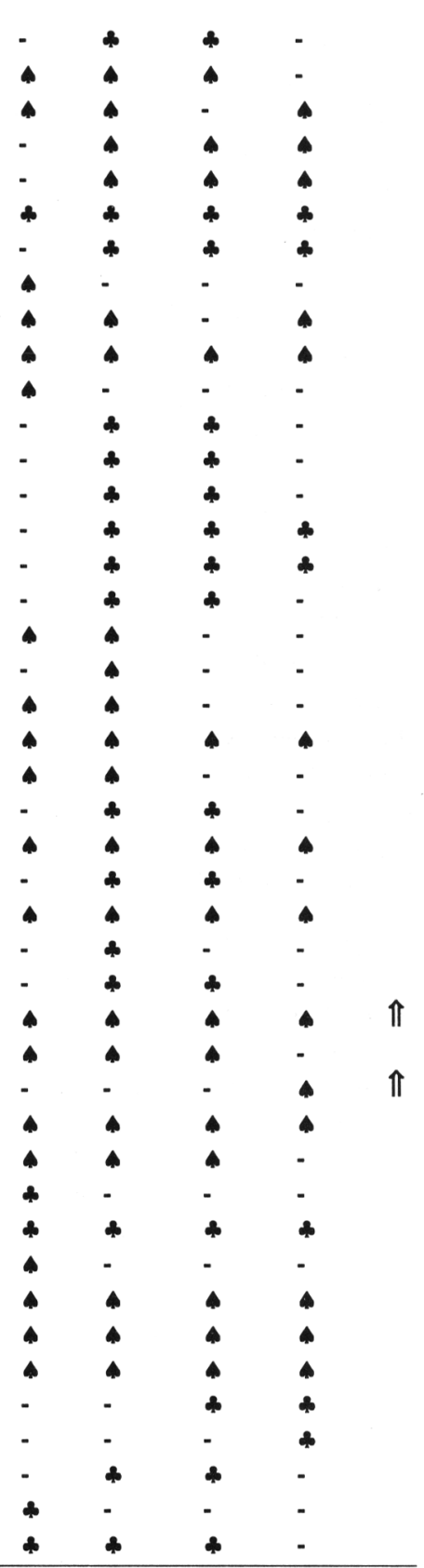

Tabla 3. Endemismos béticos y subbéticos presentes en cada subsector. \$: Endemismo subbético. \$: Endemismo bético. $\Uparrow$ : Se presenta con carácter puntual en territorios adyacentes a la provincia Bética formando parte de comunidades no béticas. Betic and Subbetic endemisms present at each subsector. \&: Subbetic endemism. \$: Betic endemism. $\Uparrow:$ Punctually present at neighbouring territories to Betic Province being found at non-Betic communities. 
subbéticas se manifiesta en el contingente florístico compartido con estos territorios. Entre los taxones de este subsector destacan, además de los ocho endemismos exclusivos, muchos elementos béticos que sólo alcanzan aquí el territorio subbético (Helictotrichon sarracenorum, Saxifraga erioblasta, Thymus granatensis, etc.). Este es uno de los rasgos más notables de este sector junto al hecho de que son, comparativamente, pocos los elementos subbéticos compartidos con los otros subsectores. Además de algunas asociaciones termófilas que dejan sentir la influencia del Valle del Guadalquivir (ClematidiCeratonietum siliquae subass. phlomidetosum purpureae, Teucrio-Coridothymetum capitatae), destaca la originalidad de este territorio en lo que a comunidades rupícolas (Sileno-Saxifragetum camposii, SarcocapnoCentauretum clementei) y de gleras (CrepidiIberidetum granatensis subass. vicietosum giennensis) se refiere, sin olvidar los tomillares dolomitícolas (Helianthemo-Pterocephaletum spatulatae). Desde el punto de vista climático es el menos lluvioso en verano, mientras que ocurre lo contrario en invierno. En conjunto destaca su marcada oposición al subsector Subbético-Murciano.

\section{Subsector Alcaracino-Cazorlense}

Las elevaciones montañosas que, mayoritariamente, incluye este nuevo subsector se disponen de una forma mucho más apretada que en el anterior. Este rasgo, sin duda, contribuye a la compacidad corológica de los territorios alcaracenses y cazorlenses. Quedan englobadas en esta unidad biogeográfica los macizos de Cazorla, El Pozo, Castril, La Cabrilla, La Sagra, Las Cabras, Guillimona, Segura y Alcaraz. Desde el punto de vista litológico, se presenta claramente delimitado frente a los materiales blandos hispalenses y guadiciano-bacenses por el predominio de las calizas y calizo-dolomías. Extraordinariamente rico en endemismos, unos 23 exclusivos, se separa de las áreas subbético-murcianas no sólo por estos elementos florísticos, sino también por múltiples rasgos de su vegetación y clima. En el primer caso destacan los bosques y orlas espinosas (Geo-Coryletum avellanae, Berberido-Quercetum pyrenaicae, Viburno lantanae-Berberidetum, etc.), matorrales (Scorzonero-Pterocephaletum spathulatae, etc.), gleras (Rumici-Aquilegietum cazorlensis, etc.), roquedos (Jasiono-Saxifragentum rigoi s. str.) y comunidades hidrófilas (PeucedanoMolinietum arundinaceae, etc.). Estos criterios pueden aplicarse también a su distinción frente al subsector Subbético-Maginense, del que queda separado por el valle del Guadiana Menor, frontera meridional para taxones como Centaurea jaennensis, Saxifraga rigoi, etc. Uno de los rasgos climáticos más acentuados en este territorio, además de la elevada cuantía de las precipitaciones en algunos puntos, 10 representa su posición de tránsito entre las áreas subbético-maginenses y las subbéticomurcianas. La separación subsectorial entre los territorios alcaracenses y segureño-

\begin{tabular}{lccccc}
\hline & SMa & Caz & Alc & SMu & $\begin{array}{c}\text { Total Sector } \\
\text { Subbético }\end{array}$ \\
\hline Bético + Subbético & 81 & 105 & 82 & 51 & 134 \\
Bético & 64 & 67 & 50 & 37 & 83 \\
Subbético & 17 & 38 & 32 & 14 & 51 \\
Exclusivos & 8 & 7 & 2 & 1 & \\
\hline
\end{tabular}

Tabla 4. Número de endemismos béticos y subbéticos presentes en cada subsector. Number of Betic and Subbetic endemisms at each subsector. 


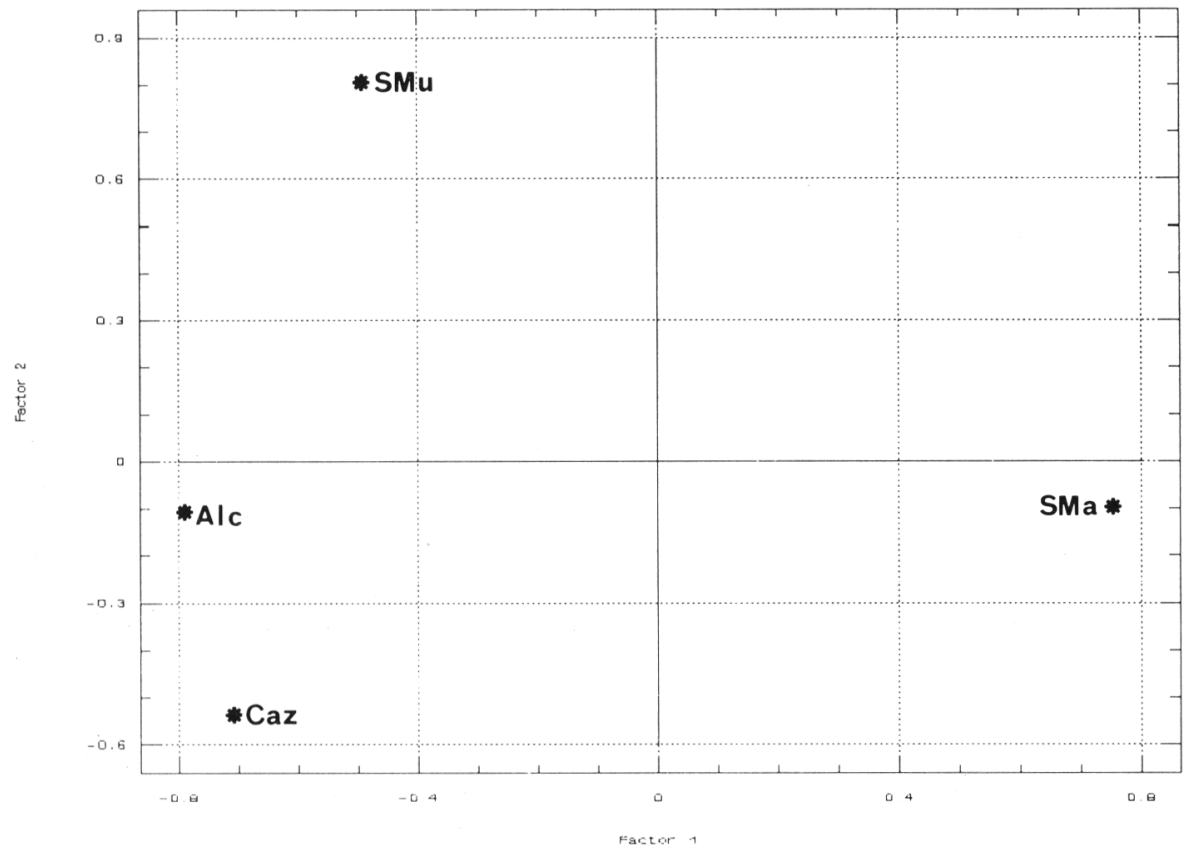

Figura 2. Análisis corológico con los dos primeros factores. Chrorologycal analysis. Principal Components 1 and 2.

Subbético-Maginense

EXCLUSIVOS

Cazorlense

8

Alcaracense

7

Subbético-Murciano

2

COMPARTIDOS POR 2 SUBSECTORES

Subbético-Maginense + Cazorlense

Cazorlense + Alcaracense

2

Cazorlense + Subbético-Murciano

14

Alcaracense + Subbético-Murciano

1

COMPARTIDOS POR 3 SUBSECTORES

Subbético-Maginense + Cazorlense + Alcaracense

Cazorlense + Alcaracense + Subbético-Murciano

4

7

\section{COMPARTIDOS POR LOS 4 SUBSECTORES}

Sub-Maginense + Alcaracense + Cazorlense + Sub-Murc.

TOTAL

Tabla 5. Número de endemismos subbéticos presentes en cada subsector o compartidos por distintos subsectores. Number of Subbetic endemisms at each subsector or shared by several subsectors. 


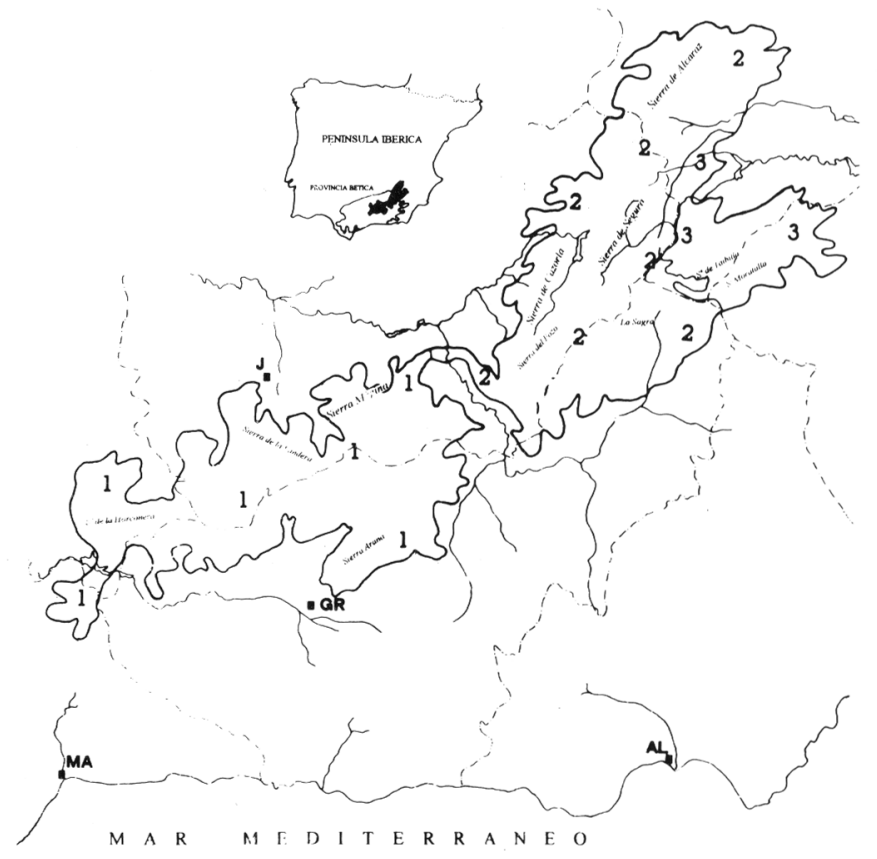

Mapa 1. Sector Subbético: 1:Subsector Subbético-Maginense. 2: Subsector Alcaracino-Cazorlense. 3: Subsector Subbético-Murciano. Subbetic Sector: 1: Subbetic-Maginense Subsector. 2: AlcaracinoCazorlense Subsector. 3: Subbetic-Murciano Subsector.

cazorlenses, parece, a la luz de los datos que se aportan, poco justificada, ya que ni existe discontinuidad paisajística ni florística. Muchos de los elementos diferenciales de la tabla 3 son muy puntuales (Geranium cazorlense, Aquilegia cazorlensis), mientras que los endemismos subbéticos compartidos son un número importante (14), tratándose con frecuencia de elementos con amplia distribución y presencia constante dentro de estos territorios como Saxifraga rigoi, Scabiosa andryalifolia, Sideritis laxespicata, etc. No es desdeñable el contacto en la parte noroccidental con la provincia Luso-Extremadurense (Sector Mariano-Monchiquense) presentando incluso de forma aislada materiales silíceos, que permiten la presencia de madroñales con brezos de óptimo luso-extremadurense, empobrecidos en la zona de contacto con el subsector Subbético-Murciano (Valle del rio Tus). En su contacto con los territorios manchegos (Sector Manchego-Guadianés), sus límites se difuminan a lo largo del Campo de Montiel.

\section{Subsector Subbético-Murciano}

Incluye las elevaciones montañosas de las Sierras de Moratalla, Zacatín, Calar de la Peña del Aguila, norte de Taibilla, las adyacentes al río Segura (Nerpio, Yeste) y las áreas próximas al río Zumeta. Es la zona más continental, lo que se plasma en algunas series de vegetación iberolevantinas, como en la que la sabina albar desempeña un papel destacable bajo una subasociación particular (Junipereto phoeniceo thuriferae S. pinetoso clusianaes.). El régimen de precipitaciones, de carácter iberolevantino, refrendado por el elevado número de taxones con esta procedencia, es claramente diferente del imperante en los otros 
subsectores, lo que se manifiesta con máximos de precipitación en primavera-otoño. Claramente separado del sector SubbéticoMaginense, presenta sin embargo algunos elementos y comunidades subbéticos compartidos con el sector AlcaracinoCazorlense especialmente con la zona más oriental (antiguo subsector Alcaracense). Son peculiares los matorrales de Salvio-Teucrietum leonis, o la vegetación dolomitícola de FumanoThymetum sabulicuae s. str. En la parte más oriental del subsector, existen incluso grandes afinidades con el sector Setabense (Provincia Valenciano-Catalano-Provenzal) con elementos tales como Lathyrus tremolsianus, Linaria cavanillesii, Erodium saxatile, etc. o comunidades incluídas dentro de la alianza Jasionion foliosae. No obstante, la presencia de gran número de taxones béticos y subbéticos así como de sintaxones (cabezas de serie) como Daphno-Pinetum sylvestris, Dahpno-Aceretum granatensis, Berberido-Quercetum rotundifoliae, Paeonio-Quercetum rotundifoliae, etc., no dejan lugar a dudas sobre su carácter bético.

\section{BIBLIOGRAFÍA}

CASTROVIEJO, S., M. LAINZ, LÓPEZ GONZÁLEZ, P. MONTSERRAT, F. MUÑOZ GARMENDIA, J. PAIVA y L. VILLAR (eds.) 1986/1994- Flora Ibérica I-IV. Real Jardín Botánico. C.S.I.C. Madrid.

CHARPIN, A. \& J. FERNÁNDEZ-CASAS -1975Plantae per Granatense Regnum et confinia lectae. Candollea, 30:43-61.

CHARPIN, A. \& J. FERNÁNDEZ-CASAS -1978Plantae per Granatense Regnum et confinia lectae. Pars altera. Candollea, 33:23-28.

ESCUDERO, A. y S. PAJARÓN -1994- Una planta nueva de la Sierra de Segura (España), Succisella andreae-molinae, sp. nov. (Dipsacaceae).Anal. Jard. Bot. Madrid, 51(2):249-254.

GOMEZ-MERCADO, F. y F. VALLE - 1990- Notas fitosociológicas sobre las comunidades arbóreas de las Sierras de Cazorla y Segura. Acta Botanica
Malacitana, 15:239-246.

GOMEZ-MERCADO, F. -1989- Cartografía y estudio de la vegetación de la Sierra de Cazorla. Tesis Doctoral. Universidad de Granada. 566 pp.

GÜEMES, J., I. MATEU y P. SÁNCHEZ-GÓMEZ -1994- Antirrhinum subbaeticum Güemes, Mateu \& Sánchez-Gómez (Scrophulariaceae), especie nueva de la Península Ibérica. Anal. Jard. Bot. Madrid, 51(2):237-247.

HERRANZ, J.M., J.J. MARTÍNEZ-SÁNCHEZ y J. DE LAS HERAS -1993-. Aportación al conocimiento del endemismo ibérico e iberoafricanismo de la flora vascular de la provincia de Albacete (España). I. Ecología, 7:179-201.

HERRANZ, J.M. -1984- El elemento endémico e iberoafricano de la flora de la Comarca de Alcaraz (Albacete). Boletín de la Estación Central de Ecología, 30:25-39.

I.N.M. Datos climáticos de Andalucía (período 1951-1989).

MOTA, J.F., F. VALLE y J. CABELLO -1993Dolomitic vegetation of South Spain. Vegetatio, 109: 29-45.

MOTA, J.F., F. GÓMEZ-MERCADO y F. VALLE1991 - Rupicolous vegetation of the betic ranges (South Spain). Vegetatio, 94:101-113.

MOTA, J.F. -1990- Estudio fitosociológico de las altas montañas calcáreas de Andalucía (Provincia corológica Bética). Tesis Doctoral. Universidad de Granada. 411 pp.

NIETO CALDERA, J.M., A.V. PÉREZ LATORRE y B. CABEZUDO -1991- Biogeografía y series de vegetación de la Provincia de Málaga (España). Acta Botanica Malacitana, 16 (2): 417-436.

RIVAS-MARTÍNEZ, S., A. ASENSI, J. MOLERO MESA y F. VALLE -1991- Endemismos vasculares de Andalucía. Rivasgodaya, 6:5-76.

RIVAS-MARTÍNEZ, S. -1987-Mapa de Series de Vegetación de España escala 1:400.000 y Memoria. I.C.O.N.A. Madrid.

RIVAS-MARTÍNEZ, S. -1993- Bases para una nueva clasificación bioclimática de la Tierra. Folia Botanica Matritensis 10.

SÁNCHEZ-GÓMEZ, P. -1990- Estudio de la Flora, Vegetación y Paisaje Vegetal de las Sierras de Segura Orientales (Albacete-Murcia). Tesis Doctoral. Universidad de Murcia. 731 pp. 
SÁNCHEZ-GÓMEZ, P y F. ALCARAZ -1992-.

Novedades fitosociológicas presentes en el subsector Subbético-Murciano (Provincia Bética) (España). Anales de Biología 18: 121152

SÁNCHEZ-GÓMEZ, P. y F. ALCARAZ -1993-. Estudio de la flora. vegetación y paisaje vegetal de las Sierras de Segura Orientales (Albacete, Murcia). Instituto de Estudios Albacetenses. Servicio de publicaciones. Albacete.459 pp. Murcia.

SÁNCHEZ-GÓMEZ, P., F. ALCARAZ y F. SAEZ 1992-. Caracterización del subsector SubbéticoMurciano (Provincia Bética). Anales de Biología, 18: 103-119.

SÁNCHEZ-GÓMEZ, P., F. SAEZ y G. LÓPEZVÉLEZ -1991- Endemismos vasculares presentes en el Suroeste de Albacete (I). AlBasit, 29:61-83.
VALLE, F., F. GÓMEZ-MERCADO y J.F. MOTA 1989- Los robledales de la Sierra de Segura y otras comunidades relacionadas con ellos. Anal. Jard. Bot. Madrid, 45(1):247-257.

Aceptado para su publicación en Junio de 1994

Dirección de los autores. P. Sánchez Gómez y F. Sáez Soto: Dpto. de Biología Vegetal (Botánica). Fac. de Biología. Universidad de Murcia.30.100Campus de Espinardo (Murcia). J.F. Mota Poveda y F. Gómez Mercado: Dpto. de Biología Vegetal. Escuela Politécnica Superior de Almería. Universidad de Almería.4120. Almería. 